source of information tapped in order that nothing may be overlooked. The annual report of the Institute is made from these records and the scientific problems which are worked out by the members of the staff are, as a rule, based on the data furnished by them. The institute has now records, - thus most laboriously and painstakingly compiled-of nearly five thousand cases.

This fourth report affords much valuable information to those interested in the problems of tuberculosis and throws light on many sideissues which have never been touched by previous observers. One of the strangest of these comes out in the comparison of the prevalence and mortality of tuberculosis in the male and female.

'The male is to a much greater extent-probably two-fold-an out-door creature than the female yet the relative mortality is 211 per hundred thousand in males and 164 per hundred thousand females. The deduction from these figures appears to be that physical hardship is perhaps a more potent factor in the developmect of tuberculosis than is the want of outdoor life. Another curious result recorded is the exceeding high mortality rate of patients whose mother was of Irish descent, while the native of native parentage of the United States appears to be less susceptible to tuberculosis than is the native of foreign parentage or the foreigner of foreign parentage. Much interesting work has been done on the age incidence of tuberculosis; the prevalence of the disease in the married and single, the question of the general build of the body, the physiognomy, appearance, complexion, etc., on the susceptibility to tuberculosis. None of these factors seem to be of much importance, the mortality was greater in patients of light complexion with light eyes than in those with dark complexion and dark eyes.

The analyses of the figures showing the result of occupation on the incidence of tuberculosis are of special interest and tend to confirm the view that hardship and deprivation are two important factors while out-door and in-door life seem to play a secondary part to these. The relative position of the occupation is undoubtedly influenced also by such factors as dust, dissipation, and low wages. The clerk and book-keeper, who are usually looked upon as the legitimate prey of consumption, stand quite low on the table of incidence; and the business man who leads an in-door life stands next to the policeman who is lowest of all on the list.

In fact all occupations which give a fair compensation and are not accompanied by severe physical labour have a relatively low incidence of tuberculosis.

The effects of alcohol and tobacco on the incidence of tuberculosis formed another subject of enquiry. 'The alcoholic with tubercle on coming under treatment either does very badly or does remarkably well. $\mathrm{Ho}_{\mathrm{o}}$ is very prone to pneumonia and nephritis; but if he escapes these, his improvement is rapid and often phenomenal. Regarding tobacco the preposterous claims that have been made regarding its preventative action cannot be maintained; quite the contrary would seem to be the true state of the case; it would appear to have a predisposing influence from the statistics brought forward.

The report is full of the most interesting items of information concerning the most common and deadly of all diseases; we have only had space to touch on the clinical and sociological part of the report, but we can thoroughly recommend its perusal to all-even those outside the ranks of the profession.

Other subjects made a study of are:-the condition of the blood, albuminuria, the pleura, tuberculosis of the bones and joints, pathological and bacteriological report of the year, relationship of the pneumococcus to hæmorrhage in tuberculosis, etc.

The staff of the institute are to be greally commended for the splendid work they are doing and to be congratulated for this latest addition to our knowledge of a disease that touches practically every family in the universe and is, therefore, of universal interest.

\section{The Propaganda for Reform in Proprietary} Medicines.-Fifth edition, revised to September 12 th, 1908.

'THIs little work contains the cream of the reports which during the last four years have been made in the journal of the American Medical Association by the Council on Pharmacy and Chemistry of the Association. It should be in the hands of every one of our readers, for not only does it afford valuable information regarding the composition of many much vaunted proprietary medicines, thus supplementing the analyses made by Zernik and the British Medical Association's Chemist, but also gives amusing instances of the disingenuous ways of the pusher of nostrums.

We may briefly give the following formulæ, which show that it is only the cheapest materials which are used in the manufacture of nostrums, though these are sold at the highest price that the "inventors" think can possibly be screwed out of the public.

Antikamnia-used to contain in America and still contains in countries outside the purview of the American Food and Drugs Act Acetanilidi 60, Caffeine 5, Ac. Citrici 5, Sodii Bicarb 20. Now in America it containsPhenacetine (acetphenitidini) 72, Caffeini 141, Ac. citrici \& Sodii Bicarb., 14-approximately.

Phenalgin-Ácetanilidi 57, Sodii bicarb., 29, Ammon. carb., 10 ; but this last ingredient is not always present.

Cactin-is in itself inert, and the fact that scspolamine and morphine are present in the H. M. C. (hyoscine, morphine, cactin) pellets 
advertised by the Abbott Alkaloidal Company, accounts for much of the action of these pellets.

Eno's Fruit Salt-contains Sodii bicarb., 50, Sodii bitart., 15, Ac. tartarici, 35. It is thus very like ordinary seidlitz powder, but of course is more expensive.

Purgen-is simply another name for phenolphthalein. As Purgen this costs about eight times what it costs under its chemical name. Another instance of the value of a name to its inventor is Urotropin, as many of our readers may remember.

Now as instances of the disregard for truth which characterizes patent medicine-vendors above all men we may take the following:-

Lactopeptine which is vaunted to contain "the five active agents of digestion-pepsin, diastase (veg. ptyatin) pancreatin, lactic acid and hydrochloric acid-combined in the proper proportion to insure the best result." More than 90 per cent. of this nostrum is milk sugar! The amount of pepsin is very small, and there is no appreciable amount of hydrochloric acid present, while careful examination failed to reveal the presence of either pancreatin or diastase. Had these ferments been present, how they could act in the presence of the stomach acid is a mystery.

Our readers will note that Lactopeptine was exposed thirty years ago, but thanks to the commercial (and unethical) spirit shown by many medical journals in their advertisement columns, it is still "going strong." Vulgus vult decipi.

Ingluvin is stated to owe its special power of remedying dyspepsia to the fact that it contains "free glycocholic acid." The presence of this substance, however, cannot be established, nor do the anatomical relations of the fowl admit of its being present!

Atoxyl is advertised as a preparation by whose use one may administer 40 times as much arsenic as by the use of Fowler's solution. The facts are that the recommended dose of atoxyl contains but $1 \frac{1}{2}$ times the amount of arsenic in the advised dose of Fowler's solution.

Vin Mariani used to be advertised as "not a cocaine preparation"; but-since the American Food and Drugs Act was passed, it is stated that each ounce "represents $\frac{1}{10}$ th of a grain of cocaine."

\section{The Etiology and Nature of Cancerous} and other Growths -.By W. T. GIBson, A.R.C.S., London, John Bale, Sons and Danielsson Ltd., 1909.

CANCER is due to irritation. Irritation is due to sunlight, X-rays, cold, tar products especially aniline and decomposition products in the intestine especially at the flexures. Also to "neurotic" conditions-oh blessed word neurotic. Cancer is curable by causing acidosis -by starvation, or by the use of non-nitrogenous aromatic acids, e.g., cinnamic acid, or the less innocuous benzoic acid-also perhaps sulphur compounds are of use, e.g., hyposulphite of sodium.

Can anything be more simple-or silly?

\section{(0)orlespondente.}

\section{PROSTATECTOMY}

To The Editor of "The Indian Medical Gazetre."

Sir,-Regarding the "Geographical distribution of the Prostatectomy operation" as mentioned in the reports of the eight cases of Prostatectomy by Capt. E. Owen Thurston, F.R.C.S., Civil Surgeon, Gaya, in your issue for February 1909 , I beg to inform you that, during my one year's service at the Dhubri Hospital (Assam) under Capt. H. Gidney, F.R.C.S.E., I.M.S., I had the good fortune to come across three well marked cases of enlarged Prostate. Capt. Gidney has, I believe, performed seven Prostatectomies in East Bengal and Assam. The Civil Surgeon gave me orders to examine very carefully the Prostate in each and every case with a present or past history of retention of urine and it was mainly due to this that these cases were detected. I think the disease is not uncommon in Assam though this opinion of mine is based on the detection of only three cases in a year in a Sadr Hospital.

Bilashipara

JISPENSARY,

17th February, 1909.

Yours faithfully,

UPENDRA MOHAN LAHIRI, Civil Hospital Assistant.

\section{QUININE IN PREGNANCY.}

To The Editor of "The Indian Medical Gazette."

SIR,-In continuation of the correspondence that has been going on in your paper on the above subject, I venture to add the following, with a few details of the cases treated.

The fact that quinine is used to overcome uterine inertia in weak women, and as an ecbolic is always prescribed during labour, if there is no obstruction, is mainly responsible for the general impression that its use should be avoided during pregnancy as far as possible. Clinical experience, however. warrants no such fear about the administration of quinine during pregnancy. Hare, in his Practical Therapeutics, says that quinine "will not of itself cause abortion, but in nervous, hysterical women who have a tendency to abort, and whom it is necessary to give full doses of quinine during pregnancy, it may be well to combine some sedative, as one of the bromides or opium with the antiperiodic." Ghosh boldly affirms that " the notion that the administration of quinine in pregnancy is highly dangerous is a mistake. Very large doses are frequently taken without causing any ill effects. Indeed, one often sees abortion result from an unchecked fever, which might have been avoided by the timely administration of quinine." (Materia Medica and Therapeutics, 3rd Edition, page 344 .)

During the last epidemic of malaria in Bombay, a large number of inhabitants of this place suffering from malaria, came up here for a change. Most of these people stay in Bombay on the Frere Ruad and the North Fort side, the headquarters so to say of the epidemic. Among these arrivals, were a number of pregnant women, all more or less infected, who applied for treatment at the dispensaly and had to be given quinine in varying doses. Not wishing to expose any of these patients to a chance of miscarriage, I first tried arsenic in the form of Fowler's solution in full doses, but this I found in 110 way prevented the next paroxysm; in some it only checked the hei ht of fever during the next attack. Quinine was, therefore, used in all the cases, eleven in number, with a distinct curative effect in most of the cases, the pregnancy continuing uninterrupted in all but one. This patient No. 8 , who miscarried after taking only two doses of quinine sulphas of 3 grains each, had a history of four months' fever, was extremely anæmia, with a puffy face and œdema of the feet, and was having feeble uterine pains for two days previous to the administration of quinine. It is very doubtful, therefore, if quinine was the sole cause of miscarriage; it may be that it intensified the feeble uterine contractions that were previously present and thus brought on miscarriage.

The following a re the details of each case :-

(1) M. G., aged 22, 1st para. Pregnant 37 weeks. Took 8 grains of Quinine Sulphas in one dose for seven days continuously. Pregnancy uninterrupted. 